\title{
Quantitative phase imaging of erythrocytes under microfluidic constriction in a high refractive index medium reveals water content changes
}

\author{
Han Sang Park', Will J. Eldridge ${ }^{1}$, Wen-Hsuan Yang $\mathbb{1}^{2,3,4}$, Michael Crose ${ }^{1}$, Silvia Ceballos ${ }^{1}$, John D. Roback
} Jen-Tsan Ashley Chi ${ }^{2,3}$ and Adam Wax ${ }^{1}$

\begin{abstract}
Changes in the deformability of red blood cells can reveal a range of pathologies. For example, cells which have been stored for transfusion are known to exhibit progressively impaired deformability. Thus, this aspect of red blood cells has been characterized previously using a range of techniques. In this paper, we show a novel approach for examining the biophysical response of the cells with quantitative phase imaging. Specifically, optical volume changes are observed as the cells transit restrictive channels of a microfluidic chip in a high refractive index medium. The optical volume changes indicate an increase of cell's internal density, ostensibly due to water displacement. Here, we characterize these changes over time for red blood cells from two subjects. By storage day 29, a significant decrease in the magnitude of optical volume change in response to mechanical stress was witnessed. The exchange of water with the environment due to mechanical stress is seen to modulate with storage time, suggesting a potential means for studying cell storage.
\end{abstract}

\section{Introduction}

The flexibility and ability to endure mechanical stress are distinguishing characteristics of red blood cells (RBC) that contribute to their functionality as a transportation mechanism of gases between lungs and tissues ${ }^{1}$. In particular, RBCs stored for transfusion can exhibit impaired function, collectively referred to as the RBC "storage

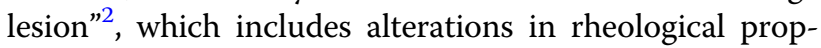
erties and physiological changes ${ }^{3,4}$. Indeed, impaired deformability of stored RBCs is a potentially serious result that affects their ability to navigate through microcirculation $^{5-7}$. Therefore, various techniques have been used to characterize the changes in RBC's rheological properties throughout the storage period $^{8-11}$. Using

Correspondence: Han Sang Park (hansang.park@duke.edu)

'Department of Biomedical Engineering, Duke University, Durham, NC 27708, USA

2Department of Molecular Genetics and Microbiology, Duke University, Durham, NC 27708, USA

Full list of author information is available at the end of the article. atomic force microscopy, previous studies have shown a significant increase in Young's modulus of the cells over the storage time indicating a decrease in the elasticity of the cells ${ }^{8,9}$. In addition, optical tweezers have been used by Czerwinska et al. to stretch RBCs and measure a significant increase in shear modulus over storage time, demonstrating a stiffer membrane ${ }^{10}$. A study by Card et al. used an ektacytometer to measure a general decrease in deformability of blood during storage by monitoring shape changes using diffractive imaging and a concentric cylinder viscometer ${ }^{11}$.

Another compelling approach for assessing RBC mechanical properties during storage is the use of microfluidic devices to quantify dynamic deformation of the cells ${ }^{12,13}$. One experiment used microfluidics with multiplexed fluidic plunger mechanisms to apply identical forces across the cells in a parallel array of funnel-shaped constrictions $^{12}$. The deformability of individual RBCs is measured based on the pressure required to push each cell through the micrometer scale constriction. Among

\section{(c) The Author(s) 2019}

(c) (i) Open Access This article is licensed under a Creative Commons Attribution 4.0 International License, which permits use, sharing, adaptation, distribution and reproduction cc) in any medium or format, as long as you give appropriate credit to the original author(s) and the source, provide a link to the Creative Commons license, and indicate if changes were made. The images or other third party material in this article are included in the article's Creative Commons license, unless indicated otherwise in a credit line to the material. If material is not included in the article's Creative Commons license and your intended use is not permitted by statutory regulation or exceeds the permitted use, you will need to obtain permission directly from the copyright holder. To view a copy of this license, visit http://creativecommons.org/licenses/by/4.0/. 
the different samples examined, there was significant variability in both the initial RBC deformability and storage capacity that were nonetheless accompanied by consistent storage-based deformability reduction. Finally, a recent study measured the stiffness of RBCs by imaging their deformation in the folding mode as they were flowing through microchannels ${ }^{13}$. Their results showed significant increases in stiffness over storage with accelerated stiffness degradation in the last 3 weeks relative to the first 3 weeks.

Here, we propose an alternative method to assess the changes in RBCs associated with storage lesion by flowing them through constricted microfluidic channels and observing nanoscale deformation with quantitative phase microscopy (QPM). QPM is a holographic method of cell imaging with nanometer path length sensitivity and the ability to observe sub-millisecond time scales ${ }^{14}$. Due to their homogeneous structure and uniform refractive index, RBCs have been a natural target for numerous studies using $\mathrm{QPM}^{15-19}$. One recent study used tomographic QPM to study changes in refractive index maps of RBCs infected with malaria parasites ${ }^{15}$. Studies from our group have used QPM to classify malaria infection with high accuracy using machine-learning algorithms ${ }^{16}$ and to quantify hemoglobin consumption by the parasite using spectroscopic information ${ }^{17}$. Another utility of QPM for RBC studies is to measure time series and observe membrane fluctuations. Our group used this approach to show reduced fluctuations of sickle cells relative to healthy $\mathrm{RBCs}^{18}$. Similarly, another independent study showed progressive reduction in temporal membrane fluctuations of stored blood over a 6-week period indicating an increase in stiffness ${ }^{19}$. QPM has been previously applied to studies involving microfluidic devices as well. Recent studies associated with microfluidic mixing ${ }^{20}$ and sperm selection ${ }^{21}$ show that these microchips can be integrated into a QPM system to quantify dynamic changes. Additionally, another group of researchers utilized the random rolling motions of the cells in microfluidic channels to obtain tomographic projections without mechanical scanning 22 .

In this paper, we present a quantitative analysis method for examining the biophysical response of RBCs as they are subjected to mechanical stress by flowing through constricted channels of custom-fabricated microfluidic chips and imaging these cells with QPM. Specifically, QPM is used to measure optical volume (OV) changes as the cells squeeze through the narrow channels. Unlike previous methods that have studied the rheological properties of the cells, we are able to observe efflux of water through the membrane of the cells under mechanical stress by using a high refractive index medium with QPM. Then, to illustrate the potential utility of this platform, the OV changes in response to mechanical force are compared for different storage periods to evaluate the changes in response to deformation that occur over the storage period.

\section{Materials and methods \\ Blood collection and preparation}

Following approval by the Institutional Review Board at Emory University, informed consent was obtained from two healthy donors. As previously described ${ }^{23}$, one unit of whole blood $(500 \mathrm{~mL} \pm 10 \%)$ was collected from each donor into CPD-ADSOL [AS-1] collection sets (Fenwal Lnc. Cat no. 4R3329E) and leukoreduced by an Integral SEPACELL ${ }^{\mathrm{TM}}$ RS-2000 filter. The filtered units of blood were then centrifuged at $3400 \mathrm{rpm}$ for $20 \mathrm{~min}$ and the plasma was replaced with ADSOL [AS-1] additive solution. These processed packed $\mathrm{RBC}$ units, which have $99.99+\%$ of $\mathrm{WBCs}$ and $~ 80 \%$ of plasma removed, were stored at $1-6^{\circ} \mathrm{C}$ for up to 42 days. Removal of aliquots of stored $\mathrm{RBC}$ was accomplished anaerobically via a steriledocking device fitted with a valve to ensure there was no re-entry of air, or other contamination, into the RBC unit. Then, the packed RBC was diluted to $0.02 \%$ hematocrit solution in $5 \mathrm{~mL}$ of a high refractive index medium composed of $20 \%$ bovine serum albumin (Sigma-Aldrich) in DPBS $+/+$ (Gibco). The refractive index of the medium was measured to be 1.372 at $23^{\circ} \mathrm{C}$ using a commercial refractometer (Bellingham + Stanley). The diluted solution was then loaded on a syringe pump system (New Era syringe pump) operating at $1.5 \mu \mathrm{L} / \mathrm{min}$ for the squeeze experiment.

\section{Development of microfluidic channels}

Glass coverslips (VWR \#1.5, $22 \times 40 \mathrm{~mm}$ ) are cleaned and dried before applying SU-8 coating. First, the substrates are exposed to a piranha cleaning process for $10 \mathrm{~min}$ followed by rinsing with acetone and isopropanol. Then, the coverslips are dehydrated at $180^{\circ} \mathrm{C}$ for $20 \mathrm{~min}$ on top of a hot plate. In order to achieve a $3 \mu \mathrm{m}$-thick coating, the coverslips are spin coated with SU8-2 (Microchem) in a headway spin coater using the following steps: SU-8 is poured on the clean substrate and distributed evenly across by spinning at $500 \mathrm{rpm}$ for $5 \mathrm{~s}$, followed by a spinning cycle at $1030 \mathrm{rpm}$ for $45 \mathrm{~s}$. For presoft bake, the exposed substrates are heated on top of a hotplate at $65^{\circ} \mathrm{C}$ for $1 \mathrm{~min}, 95^{\circ} \mathrm{C}$ for $3 \mathrm{~min}$, and back to $65^{\circ} \mathrm{C}$ for $1 \mathrm{~min}$. The exposure is performed on a Karl Suss MA6 mask aligner using a UV lamp with an exposure intensity of $84 \mathrm{~mJ} / \mathrm{cm}^{2}$. The pattern mask used for the exposure is shown in Fig. 1a. The post exposure bake is $65^{\circ} \mathrm{C}$ for $1 \mathrm{~min}$ followed by $95^{\circ} \mathrm{C}$ for 1 min and back to $65^{\circ} \mathrm{C}$ for $1 \mathrm{~min}$ on top of a hotplate. The resist is developed in SU-8 developer for $1 \mathrm{~min}$, then rinsed with isopropanol and blow-dried with $\mathrm{N}_{2}$. Using a profilometer (Bruker Dektak 150), the height of the developed 

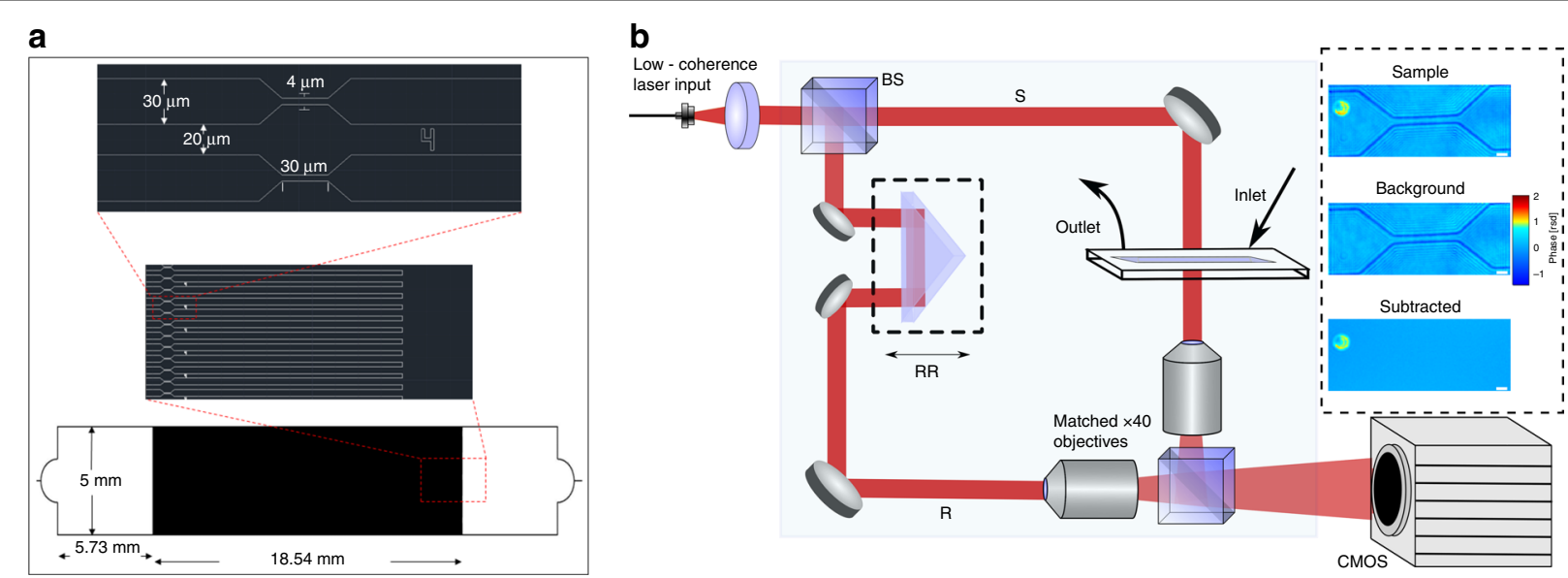

Fig. 1 System setup. a Schematics of pattern mask used for photolithography. b Quantitative phase microscopy system: beam splitter (BS), retroreflector (RR). Path-matched sample (S) and reference (R) beams create off-axis interferograms imaged by a CMOS camera. Inset: Phase images of the sample, the background, and the RBC after background subtraction. Scale bar $=5 \mu \mathrm{m}$.

photoresists is measured to be $3.222+0.109 \mu \mathrm{m}$. In order to enable mechanical stress to be applied to RBCs, the developed pattern has 100 openings that lead to six constriction areas each with $4 \mu \mathrm{m}$ width. The fabricated channels are covered with glass slides $(\mathrm{VWR}, 25 \times 75 \times$ $1 \mathrm{~mm}$ ) with both inlets and outlets created using a drill press with a diamond bit. In order to bond the cover glass to the SU-8 channels, a chamber with a customized manual press using bolted joint design ${ }^{24}$ is used to apply controlled pressure $(2.11 \mathrm{MPa})$ with a torque wrench. While the microfluidic device is under pressure, it is heated in a convection oven at $180^{\circ} \mathrm{C}$ for $1 \mathrm{~h}$. Finally, blocks of polydimethylsiloxane with punched holes are bonded to the inlet and outlet of the devices using a plasma asher (Trion Technology Phantom II) to hold the tubing.

\section{Quantitative phase microscopy}

Our custom quantitative phase microscopy system (QPM, Fig. $1 \mathrm{~b})^{25}$ is used to image the RBCs flowing through the microchannels. Using a customized spectral filter, broadband light from a supercontinuum laser source (Fianium SC-400-4) is filtered to a $1.12 \mathrm{~nm}$ fullwidth at half-maximum spectral bandwidth with a center wavelength at $680 \mathrm{~nm}$ (coherence length, $l_{\mathrm{c}}: 204 \mu \mathrm{m}$ ).

Light at the selected wavelength is coupled into a singlemode fiber and transmitted to the off-axis Mach-Zehnder interferometer as a collimated beam. In the interferometer, the illumination light is separated by a beam splitter into sample and reference arms which are then path-matched to within the coherence length of the filtered light using mirror-based retroreflectors on translation stages. Matched microscope objectives (Zeiss PlanNeoFLUAR $\times 400.75 \mathrm{NA}$ ) are used in each arm, creating an image of the sample with an effective magnification of $\sim$ x107. The CMOS camera (Photron FastCam SA-4, $1024 \times 1024$ px, 10-bit data capture) detects the off-axis interferogram created by the angle difference between the reference and the sample arms.

In order to produce quantitative phase images, $\Delta \phi(x, y)$, the interferograms are digitally processed as described in previous works ${ }^{16,26}$. The acquired interferograms are Fourier transformed to isolate one of the complex conjugate components by spatially filtering around the carrier frequency. Then, the filtered spatial frequency information is demodulated and inverse Fourier transformed to produce an image of the complex field containing both amplitude and phase information. Finally, the phase image is obtained by taking the angle of the complex field at each spatial location.

Since QPM obtains the complex optical field (amplitude and phase), the image can be digitally propagated to ensure they are in focus. This is an important aspect since incorrect focus can produce erroneous analysis ${ }^{27}$. After subtracting background noise, using a background field of view (FOV) image without any cells, the images of flowing RBCs (inset of Fig. 1b) are digitally refocused to an optimal distance as determined using the minimum variance of amplitude of the RBC for the first three frames. Then, to ensure the correct focal plane has been found, the area of the cell is calculated for each frame during its flow through the constricted channel over a range of propagation distances to determine its minimum value. The offset propagation distance found by determining the minimum area from the distance determined using minimum variance of amplitude is in agreement with results from our previous paper that examined digital focusing during stress-free flow through a channel ${ }^{28}$. 

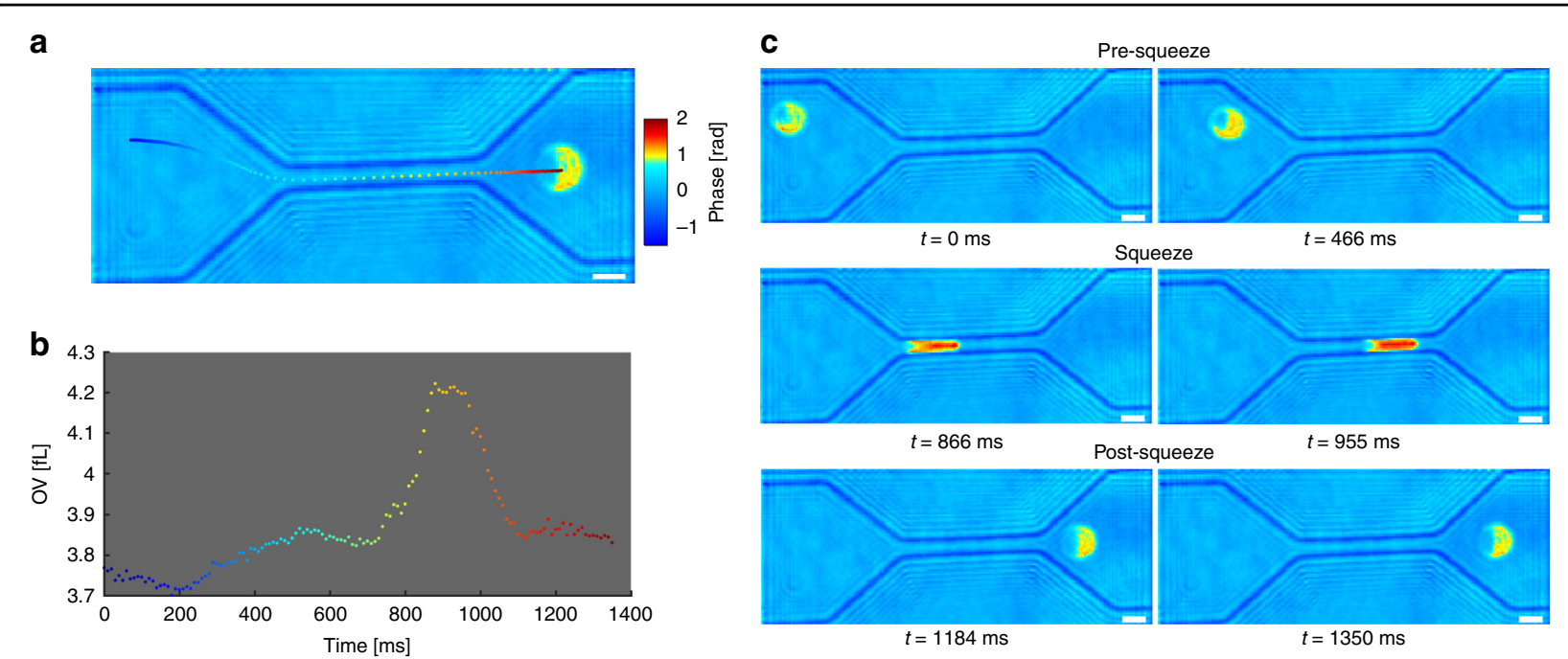

Fig. 2 Transit through a microfluidic channel. a An RBC flowing through a channel while its position throughout the flow is color-coded to correspond to the temporal OV change shown in b. c Three different segments (pre-squeeze, squeeze, and post-squeeze) of the transit through the constricting channel. Scale bar $=5 \mu \mathrm{m}$ (see Mov 1 for video).

After subtracting the background FOV that is corrected for defocus using the same determined propagation distance, any remaining phase noise only varies slowly across the field of view, arising from the temporal drift between the two interferometer arms. It is removed by fitting the background, excluding the RBCs, to third-order polynomials and subtracting. Then, changes in optical path length (OPL) of RBCs are calculated as

$$
\Delta \mathrm{OPL}(x, y, t)=\Delta n(x, y, t) \cdot h(x, y, t)=\Delta \phi(x, y, t) \cdot \lambda / 2 \pi
$$

where $h(x, y, t)$ is the height map of the object and $\Delta n(x, y, t)$ is the refractive index map. The red blood cells are automatically segmented from each FOV by applying both optical path length and area thresholds from previous literature ${ }^{29}$. Also, in order to measure the volume changes to the $\mathrm{RBC}$ as it transits through the constricted channels, the $\triangle \mathrm{OPL}$ is integrated to obtain optical volume $\left(\mathrm{OV}^{27}\right)$ :

$$
\mathrm{OV}(t)=\iint_{x, y} \Delta \mathrm{OPL}(x, y, t) \mathrm{d} x \mathrm{~d} y=\iint_{x, y} \Delta n(x, y, t) \cdot h(x, y, t) \mathrm{d} x \mathrm{~d} y
$$

In our previous studies evaluating OV, we showed that this parameter was invariant for RBCs as they change orientation ${ }^{28,30}$. These studies examined cells over periods of $10 \mathrm{~s}$ or longer and showed that OV did not change by more than $2 \%$ over this period.

An example of the transit through the constricted channel as well as corresponding OV measurements throughout the flow are shown in Fig. 2a, b. In order to quantify volume changes during the flow, total transit time is manually divided into three different segments as pre-squeeze, squeeze, and post-squeeze as shown in Fig. 2c. The three segments are selected by ensuring that the cells are not mechanically stressed nor in contact with the walls during the pre-sqeeze and post-squeeze segment and making sure that the entire cell is within the constricting channel during the squeezing segment.

\section{Results}

In order to observe the biophysical changes to RBCs over the storage period, two different blood samples are imaged at three time points: day 1, 15, and 29. The number of cells imaged on each day for the two samples are shown in Table 1.

As can be seen in Fig. 3, the OV of the RBCs between day 1 and day 15 does not show statistically significant difference throughout the three stress states for both samples. In contrast, the differences in OV between day 1 and day 29, as well as between day 15 and day 29, are statistically significant for both samples. These results arise from a general increase in $\mathrm{OV}$ at day 29 relative to the other earlier storage time points, regardless of the mechanical stress state. This trend can be seen in Table S1 in the supplementary information with the average as well as the standard deviation of the populations in Fig. 3.

An increase in $\mathrm{OV}$ is observed during the squeeze state relative to both pre-squeeze and post-squeeze state regardless of the storage day. In order to further analyze this trend at the single-cell level, the biomechanical properties of RBCs during storage are further quantified 
by calculating the percentage change in $\mathrm{OV}$ during the squeeze segment relative to the OV before entering the constricted channel (Fig. 4). The percentage change in OV between the two states, $\Delta \mathrm{OV}_{\mathrm{SP}}$, is calculated as

$$
\Delta \mathrm{OV}_{\mathrm{SP}}=\frac{\left(\overline{\mathrm{OV}}_{\text {Squeeze }}-\overline{\mathrm{OV}}_{\text {Pre }}\right)}{\overline{\mathrm{OV}}_{\text {Pre }}} \times 100
$$

Table 1 Sample size.

\begin{tabular}{llll}
\hline & Day 01 & Day 15 & Day 29 \\
\hline Sample 01 (Female, age: 69) & 66 & 95 & 94 \\
Sample 02 (Male, age: 51) & 96 & 42 & 79 \\
\hline
\end{tabular}

The boxplots of OV for the three different mechanical stress states at all the storage time points for the two samples are shown in Fig. 3a, b where $\overline{\mathrm{OV}}_{\text {Squeeze }}$ and $\overline{\mathrm{OV}}_{\text {Pre }}$ are the average $\mathrm{OV}$ of the flow segments during and before the constricted channel, respectively.

Similar to the OV comparison in Fig. 3, there is no significant difference for $\Delta O V_{S P}$ between day 1 and day 15. In contrast, there are statistically significant differences in $\Delta \mathrm{OV}_{\mathrm{SP}}$ between day 1 and day 29 as well as between day 15 and day 29. No correlation was observed between the $\Delta \mathrm{OV}_{\mathrm{SP}}$ for a given cell and its original optical volume (Supplementary Information). The average $\Delta \mathrm{OV}_{\mathrm{SP}}$ as well as the corresponding standard deviation and the observed range in values for the data in Fig. 4 are shown in Table S2 in the supplementary information.

As can be seen in Fig. 4, the positive $\Delta \mathrm{OV}_{\mathrm{SP}}$ values indicate that there is an increase in OV as the cells are squeezed through the constricted channel. Also, it should be noted from the measured data above that the $\Delta O V_{S P}$ values, as well as the corresponding standard deviation and ranges, are much larger at day 1 and 15 than those at

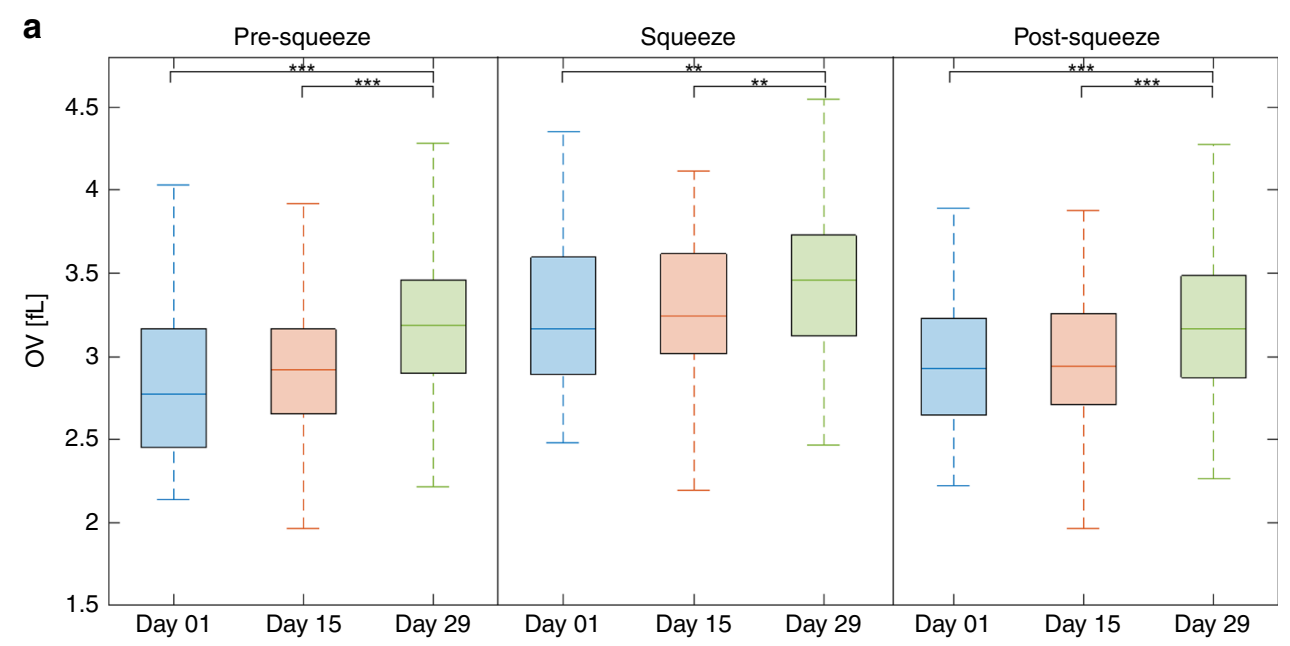

b

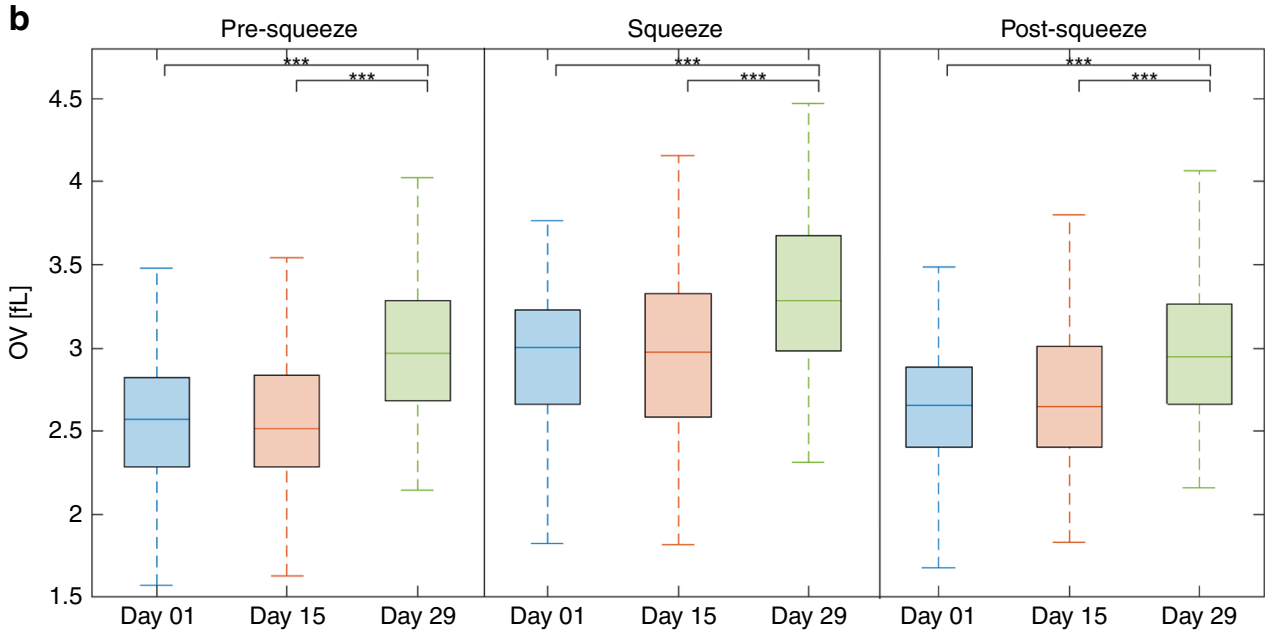

Fig. 3 Box plots of RBC's OV flowing through the microchannels. a Sample 01 and $\mathbf{b}$ Sample 02 (** for $P<0.01 ;{ }^{* *}$ for $P<0.001$ ). 


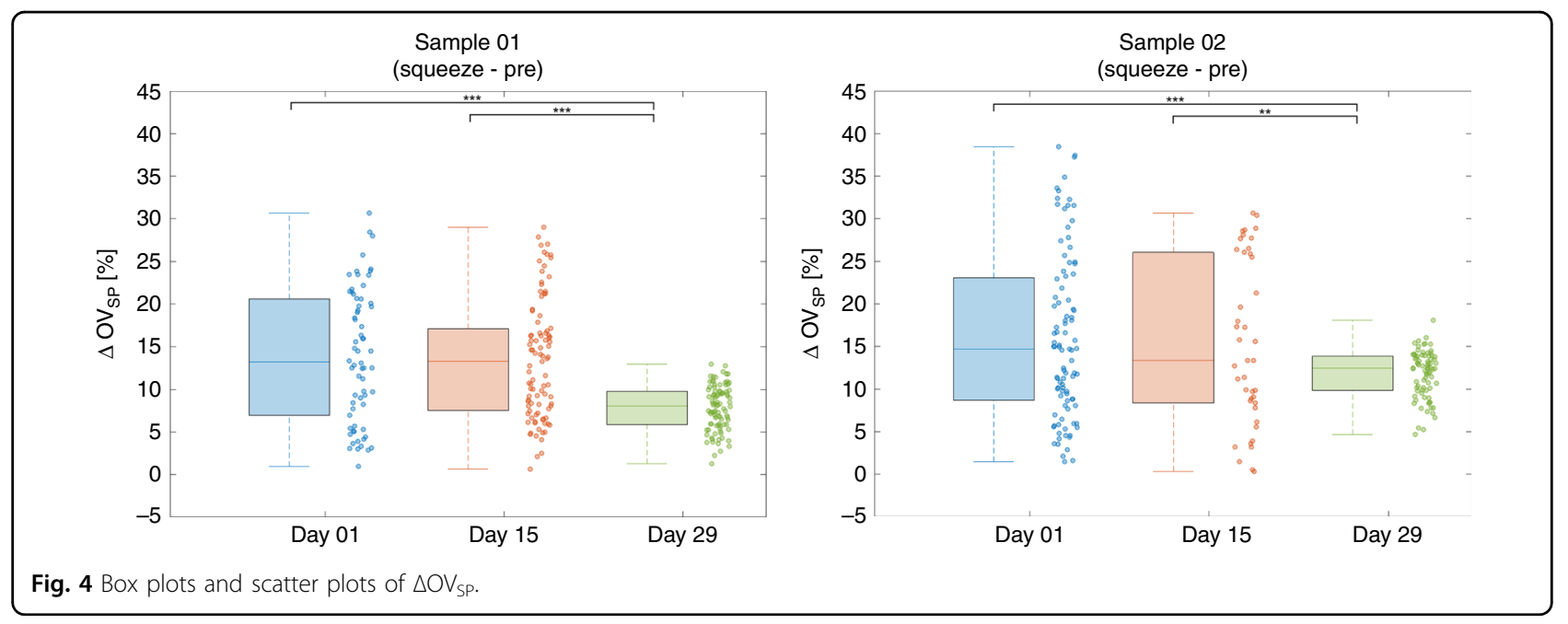

day 29 for both samples. The decrease in the variability of $\triangle \mathrm{OV}_{\mathrm{SP}}$ over storage time could be an important storagebased characteristic resulting from a population distribution shift from a mixture of younger and older cells to a population composed mostly of the older RBCs.

Further insight can be gained by examining the percentage change in $\mathrm{OV}$ after leaving the constriction channel compared to the OV before entering the channel (Fig. 5). The percentage change in OV these two states, $\Delta \mathrm{OV}_{\mathrm{PP}}$, is calculated as

$$
\Delta \mathrm{OV}_{\mathrm{PP}}=\frac{\left(\overline{\mathrm{OV}}_{\text {Post }}-\overline{\mathrm{OV}}_{\text {Pre }}\right)}{\overline{\mathrm{OV}}_{\text {Pre }}} \times 100
$$

where $\overline{\mathrm{OV}}_{\text {Post }}$ is the average OV of the flow segment after the constricted channel.

Except for the difference in $\Delta O V_{\mathrm{PP}}$ between day 1 and 15 for sample 1, there are significant differences between the other storage times. No correlation was observed between the $\Delta \mathrm{OV}_{\mathrm{PP}}$ for a given cell and its initial $\mathrm{OV}$ (Supplementary Information). The average as well as the corresponding standard deviation and the range of the populations in Fig. 5 are shown in Table S3 in the supplementary information.

The ranges of $\Delta O V_{\mathrm{PP}}$ at day 1 and 15 are larger than the $\Delta \mathrm{OV}_{\mathrm{PP}}$ at day 29 , as shown in Fig. 5, resembling the trend shown in Fig. 4 for $\Delta O V_{S P}$. It should be also noted that the average $\Delta \mathrm{OV}_{\mathrm{PP}}$ is closer to 0 at day 29 than for the other time points indicating that more cells are able to recover the original $\mathrm{OV}$ after squeezing through the channels.

\section{Discussion}

Numerous studies have examined storage-based changes in rheological properties of RBCs and have shown decreased deformability in RBCs associated with oxidative stress $^{31}$ and increased mean corpuscular hemoglobin concentration $^{32}$. In addition, the recent study by Alshalani et al. emphasized the importance of monitoring water permeability as a potential predictor to assess the quality of the RBCs during hypothermic storage ${ }^{33}$. Using QPM, we analyzed RBCs flowing through constrictive microfluidic channels after different lengths of storage time. Here, we observed changes in optical volume, which can be an indication of transport of water from the cells. This unique observation was enabled by using a high refractive index medium and a fixed channel height. Thus, when the cells were mechanically compressed, the density was seen to increase relative to the background as water was displaced. This platform could further our knowledge of the deformability of RBCs, their ability to transfer fluids with their environment and the impact of constriction during circulation through small microvasculature.

In this paper, we have examined how RBCs respond to mechanical stress. With further development, this platform could be applied to the study of stored blood units. The current Food and Drug Administration guideline for 42-day RBC storage interval is based on recovery of over $75 \%$ of transfused RBCs in circulation at a time point of $24 \mathrm{~h}$ post-transfusion ${ }^{2}$. The benchmark assumes that RBCs which remain in circulation after the 24-h window will have normal lifespans ${ }^{34}$. However, their presence in circulation alone does not necessarily indicate their ability to function as a transportation vehicle of oxygen to tissue. Since the majority of oxygen exchange occurs in the microvasculature, it is important to assess the ability of RBCs to tolerate high pressure while flowing through narrow capillaries much smaller than their own diameters ${ }^{1}$. We believe that measurement of OV changes for RBCs under mechanical forces as a function of storage time could be applicable to assessment of stored cells. 


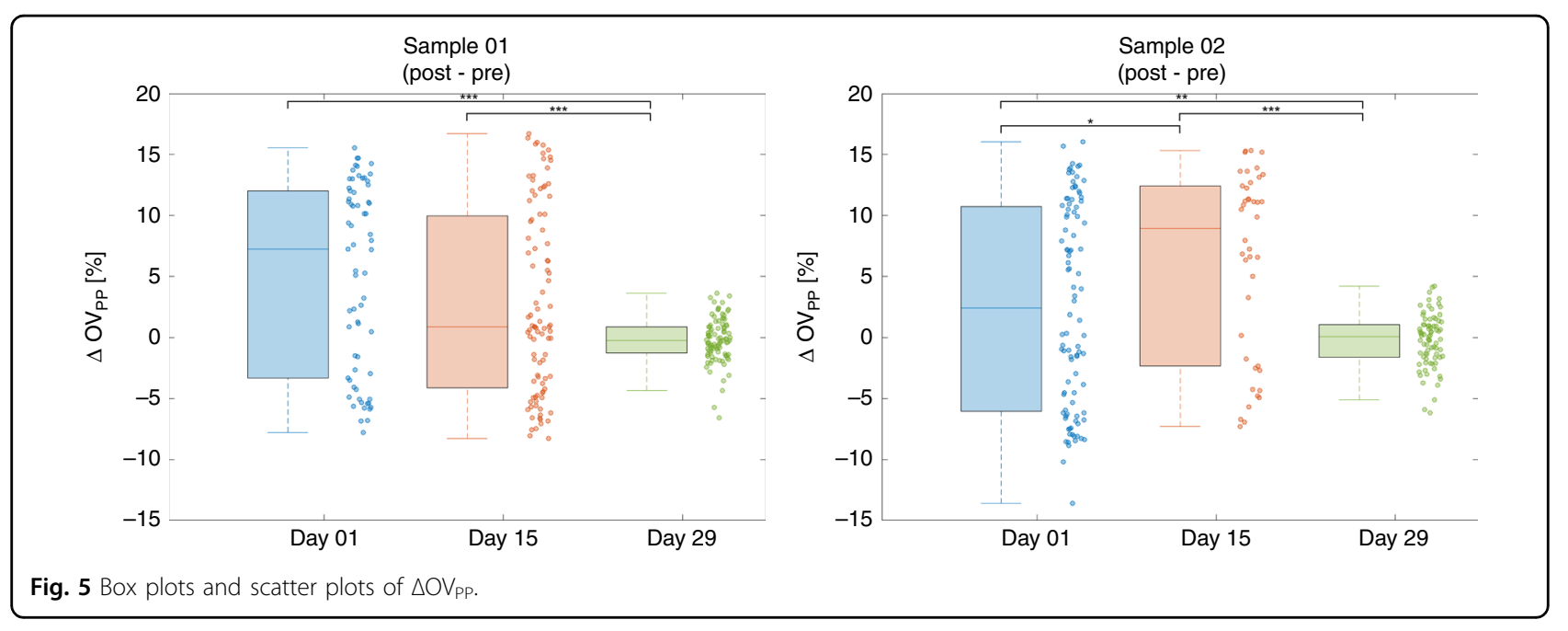

As can be seen in Fig. 3, there are statistically significant differences between the OV of the cells at day 1 and 29 as well as between those at day 15 and 29. The differences between the populations are due to an increase in OV of the cells at day 29 relative to those at day 1 and 15 . The increase in OV can be correlated to findings of a previous study by Park et al. ${ }^{35}$, where an increase in hemoglobin concentration was measured for stored RBCs. The study showed a significant increase in hemoglobin concentration over the storage period without a significant change in total hemoglobin content over the same period, an effect that could be accounted for by water efflux through the membrane. A similar increase in density throughout the lifecycle of RBCs in circulation has been reported in previous studies as well ${ }^{36-39}$.

As shown above in Eq. (2), OV is derived from both the cell's physical height as well as its refractive index difference relative to the medium. We now consider the relative contribution of each component. By assuming uniform refractive index throughout the $\mathrm{RBC}$, OV can be expressed as

$$
\mathrm{OV}(t)=\Delta \bar{n}(t) \cdot V(t)=\left(\bar{n}_{\mathrm{RBC}}(t)-n_{\mathrm{m}}\right) \cdot V(t)
$$

where $\Delta \bar{n}(t)$ is the average refractive index difference of the cell and $V(t)$ is the physical volume of the cell. Furthermore, the average refractive index difference $\Delta \bar{n}$ is derived by subtracting the refractive index of the medium used in the experiment, $n_{\mathrm{m}}$, from the average refractive index of the cell, $\bar{n}_{\mathrm{RBC}}(t)$. Therefore, the optical volume change over the storage time can be expressed as a ratio as

$$
\mathrm{OV}_{\text {ratio }}(t)=\frac{\left(\bar{n}_{\mathrm{RBC}}\left(t_{\mathrm{f}}\right)-n_{\mathrm{m}}\right)}{\left(\bar{n}_{\mathrm{RBC}}\left(t_{\mathrm{i}}\right)-n_{\mathrm{m}}\right)} \cdot \frac{V\left(t_{\mathrm{f}}\right)}{V\left(t_{\mathrm{i}}\right)}
$$

As can be seen in Eq. (6), the change in OV over time can be sensitive to the change in refractive index of the cell by selecting the refractive index of the medium, $n_{\mathrm{m}}$. By using a higher $n_{\mathrm{m}}$, closer to the refractive index of the cell rather than that of water, any volume decrease due to the displacement of water from the cell can be made to have a lesser effect than the increase in the refractive index difference due to a higher concentration of hemoglobin. In order to demonstrate the expected OV change as a function of the volume of water displaced from the cell, a RBC model was simulated using typical values from the literature (given in Supplementary Information) and is studied in Fig. 6.

As can be seen in Fig. 6a, the rate at which the refractive index difference increases due to water displacement becomes more pronounced as the medium refractive index, $n_{\mathrm{m}}$ is increased compared to that of water. Also, the physical volume $V$ decreases linearly due to water loss regardless of the choice of $n_{\mathrm{m}}$. Hence, as the refractive index of the selected medium $n_{\mathrm{m}}$ increases, the relative effect due to refractive index difference becomes greater than the effect of the reduction in physical volume due to water loss. This concept is reflected in the overall percent change in OV as shown in Fig. 6b. Therefore, the increase in OV over storage time in Fig. 3 is believed to be largely due to a change in refractive index, as a result of the efflux of water through the membrane, producing an effective net increase in hemoglobin concentration. The correlation between the water content change and the optical volume change was validated with the osmolarity experiment shown in the supplementary information. The results of the experiment showed that changes in water content of cell can be estimated using optical volume as measured with the system.

Similarly, the increase in OV during the squeeze segment of the flow, as shown in Figs. 3 and 4, can also be explained by displacement of water from the cells under stress. As they flow through the constricting channel, the RBCs experience mechanical pressure which forces them 

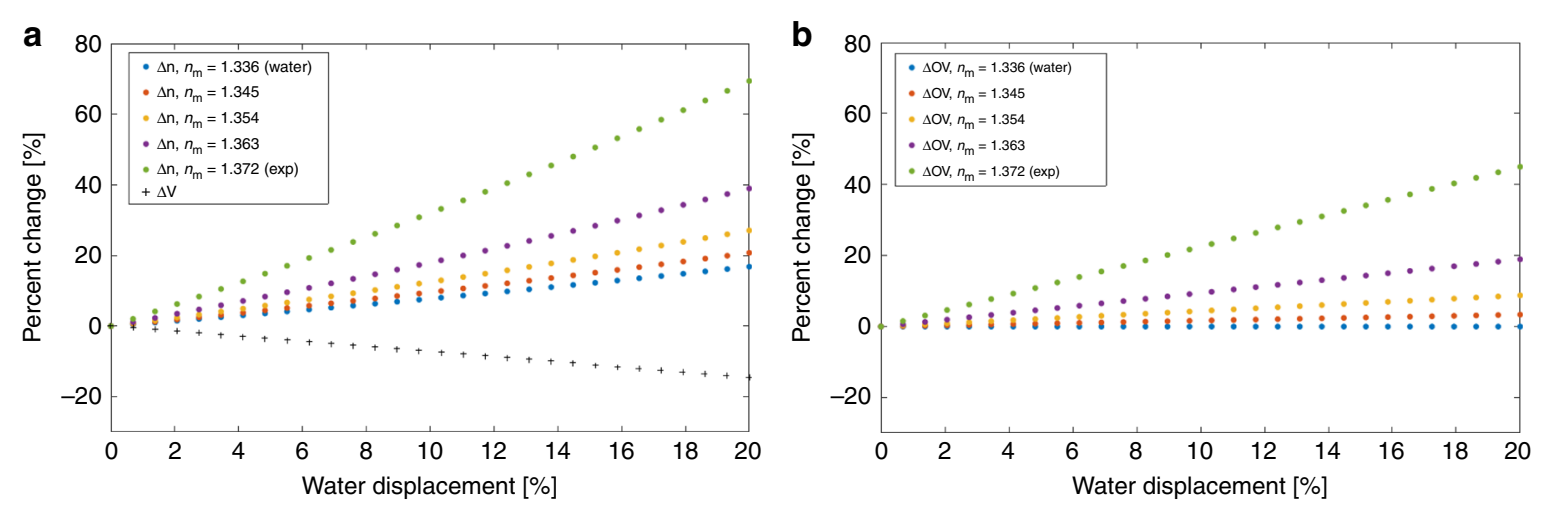

Fig. $\mathbf{6} \Delta \mathrm{OV}$ simulation. a $\Delta n$ and $\triangle V$ over displacement of water from RBC at different $n_{m}$. $\mathbf{b} \triangle O V$ over displacement of water from RBC at different $n_{\mathrm{m}}$.

to discharge water. A previous study by Gambhire et al. have also suggested possible volume loss by RBCs during their transition through constricting PDMS channels, simulating RBCs passing through inter-endothelial slits, as an explanation for the transition of RBCs with high sphericity index ${ }^{40}$. Interestingly, cells at day 1 and day 15 show a larger range of $\Delta \mathrm{OV}_{\mathrm{SP}}$ relative to the cells at day 29. This can be explained by considering that over the storage period, the population of RBCs shifts from a mixture of younger and older cells towards a population distribution consisting of largely older cells. Hence, the reduction in the range of OV change, corresponding to less water discharge under stress, could be perhaps be used as an indicator of aging RBCs during storage.

Another interesting aspect of our findings is the degree to which RBCs recover their OV after stress. The ranges of $\Delta \mathrm{OV}_{\mathrm{PP}}$, shown in Fig. 5, are larger at day 1 and day 15 than that at day 29 as the average $\Delta O V_{\mathrm{PP}}$ at day 29 is closer to 0 . Due to a general decrease in water displacement under mechanical stress at day 29 , more cells are able to recover the original OV after the transit through the constricting channels. It should be noted that a previous study by Sun et al. ${ }^{13}$ has shown significant increase in stiffness of RBCs over the storage time especially by week 4. Similarly, our experiment shows significant changes in $\Delta \mathrm{OV}_{\mathrm{SP}}$ and $\Delta \mathrm{OV}_{\mathrm{PP}}$ by day 29 , corresponding to less exchange of water with the medium by RBCs under stress. Both of these rheological properties, which directly affect cell deformability, show significant change over storage across a similar time period. In order to show a relationship between the rheological properties, RBCs were chemically degraded through glutaraldehyde treatment and imaged with the system as shown in the supplementary information (Fig. S4). There was a significant increase in OV for those cells treated with glutaraldehyde in a dose-dependent manner, possibly due to an increase in stiffness as a result of an increase in the viscosity of the cytoplasm. Further analysis of the relationship between these parameters of the cells as well as their changes over storage could have an impact on monitoring of stored blood by distinguishing older cells that may not be as effective in transiting through microvasculature.

\section{Conclusion}

In this paper, we measured OV changes of RBCs as they transit through constricting channels of microfluidic devices at different storage times using QPM. RBCs that had been stored for up to 29 days showed a general increase in $\mathrm{OV}$ corresponding to the known increase in hemoglobin concentration over the storage period. In addition, $\mathrm{OV}$ increased as the RBCs were squeezed in the narrow channels also due to higher hemoglobin concentration arising from water displacement through the membrane in response to mechanical stress. Interestingly, at day 29, significant reduction in OV changes relative to earlier time points were observed, possibly implying less water exchange by an RBC population composed of older cells. The change in the biophysical response of the cells over a storage period can impact their ability to effectively travel through narrow microvessels. The results shown here suggest that QPI in combination with constrictive microfluidics and high index media can be used to monitor unique aspects of RBC biomechanics with potential importance to evaluation and monitoring of stored blood.

\section{Acknowledgements}

Work supported by NSF (CBET-1604562), NIH (1R21 ES029791), World antidoping agent (WADA) and Partnership for Clean Competition (PCC).

\section{Author details}

${ }^{1}$ Department of Biomedical Engineering, Duke University, Durham, NC 27708, USA. ${ }^{2}$ Department of Molecular Genetics and Microbiology, Duke University, Durham, NC 27708, USA. ${ }^{3}$ Duke Center for Genomic and Computational Biology, Duke University, Durham, NC 27708, USA. ${ }^{4}$ Department of Biochemistry, Duke University, Durham, NC 27708, USA. ${ }^{5}$ Department of 
Pathology and Laboratory Medicine, Emory University School of Medicine, Atlanta, GA 30322, USA

\section{Conflict of interest}

The authors declare that they have no conflict of interest.

Supplementary information accompanies this paper at https://doi.org/ 10.1038/s41378-019-0113-y.

Received: 22 December 2018 Revised: 6 May 2019 Accepted: 15 October 2019

Published online: 02 December 2019

\section{References}

1. Zinchuk, V. V. [Erythrocyte deformability: physiological aspects]. Usp. Fiziol. Nauk 32, 66-78 (2001).

2. Zimring, J. C. Established and theoretical factors to consider in assessing the red cell storage lesion. Blood 125, 2185-2190 (2015).

3. Hess, J. R. Red cell changes during storage. Transfus. Apher. Sci. 43, 51-59 (2010).

4. Hess, J. R. Red cell storage. J. Proteom. 73, 368-373 (2010).

5. Daly, A., Raval, J. S., Waters, J. H., Yazer, M. H. \& Kameneva, M. V. Effect of blood bank storage on the rheological properties of male and female donor red blood cells. Clin. Hemorheol. Microcirc. 56, 337-345 (2014).

6. Berezina, T. L. et al. Influence of storage on red blood cell rheological properties. J. Surg. Res. 102, 6-12 (2002).

7. Yalcin, O., Ortiz, D., Tsai, A. G., Johnson, P. C. \& Cabrales, P. Microhemodynamic aberrations created by transfusion of stored blood. Transfusion 54, 1015-1027 (2014).

8. Lamzin, I. M. \& Khayrullin, R. M. The quality assessment of stored red blood cells probed using atomic-force microscopy. Anat. Res. Int. https:/doi.org/ 10.1155/2014/869683 (2014)

9. Kozlova, E. et al. Morphology, membrane nanostructure and stiffness for quality assessment of packed red blood cells. Sci. Rep. 7, 7846 (2017).

10. Czerwinska, J., Wolf, S. M., Mohammadi, H. \& Jeney, S. Red blood cell aging during storage, studied using optical tweezers experiment. Cell. Mol. Bioeng. $\mathbf{8}$, 258-266 (2015).

11. Card, R. T., Mohandas, N., Perkins, H. A. \& Shohet, S. B. Deformability of stored red blood cells. Relationship to degree of packing. Transfusion 22, 96-101 (1982).

12. Matthews, K. et al. Microfluidic deformability analysis of the red cell storage lesion. J. Biomech. 48, 4065-4072 (2015).

13. $\mathrm{Xu}, \mathrm{Z}$. et al. Stiffness increase of red blood cells during storage. Microsyst Nanoeng. 4, 17103 (2018).

14. Shaked, N. T., Rinehart, M. T. \& Wax, A. Dual-interference-channel quantitativephase microscopy of live cell dynamics. Opt. Lett. 34, 767-769 (2009).

15. Park, Y. et al. Refractive index maps and membrane dynamics of human red blood cells parasitized by Plasmodium falciparum. Proc. Natl Acad. Sci. USA 105, 13730-13735 (2008).

16. Park, H. S., Rinehart, M. T., Walzer, K. A., Chi, J.-T. A. \& Wax, A. Automated detection of $P$. falciparum using machine learning algorithms with quantitative phase images of unstained cells. PLOS ONE 11, e0163045 (2016).

17. Rinehart, M. T., Park, H. S., Walzer, K. A., Chi, J.-T. A. \& Wax, A. Hemoglobin consumption by $P$. falciparum in individual erythrocytes imaged via quantitative phase spectroscopy. Sci. Rep. 6, 24461 (2016).
18. Shaked, N. T., Satterwhite, L. L., Truskey, G. A., Wax, A. P. \& Telen, M. J. Quantitative microscopy and nanoscopy of sickle red blood cells performed by wide field digital interferometry. J. Biomed. Opt. 16, 030506 (2011).

19. Bhaduri, B., Kandel, M., Brugnara, C., Tangella, K. \& Popescu, G. Optical assay of erythrocyte function in banked blood. Sci. Rep. 4, 6211 (2014).

20. Park, G. et al. Visualization and label-free quantification of microfluidic mixing using quantitative phase imaging. Appl. Opt. 56, 6341-6347 (2017).

21. Eravuchira, P. J. et al. Individual sperm selection by microfluidics integrated with interferometric phase microscopy. Methods 136, 152-159 (2018).

22. Merola, F. et al. Tomographic flow cytometry by digital holography. Light Sci. Appl. 6, e16241 (2017).

23. Yang, W.-H. et al. Angiogenin-mediated tRNA cleavage as a novel feature of stored red blood cells. Br. J. Haematol. https://doi.org/10.1111/bjh.15605 (2018)

24. Serra, S. G. et al. A simple bonding process of SU-8 to glass to seal a microfluidic device. In Proc. 3rd International Conference on Multi-Material Micro Manufacture, Borovets, Bulgaria pp. 3-5 (2007)

25. Rinehart, M., Zhu, Y. \& Wax, A. Quantitative phase spectroscopy. Biomed. Opt Express 3, 958 (2012).

26. Liebling, M., Blu, T. \& Unser, M. Complex-wave retrieval from a single off-axis hologram. J. Opt. Soc. Am. A 21, 367 (2004).

27. Rinehart, M. T., Park, H. S. \& Wax, A. Influence of defocus on quantitative analysis of microscopic objects and individual cells with digital holography. Biomed. Opt. Express 6, 2067 (2015).

28. Park, H. S., Ceballos, S., Eldridge, W. J. \& Wax, A. Invited Article: digital refocusing in quantitative phase imaging for flowing red blood cells. APL Photonics $\mathbf{3}$, 110802 (2018).

29. Diez-Silva, M., Dao, M., Han, J., Lim, C.-T. \& Suresh, S. Shape and biomechanical characteristics of human red blood cells in health and disease. MRS Bull. Mater. Res. Soc. 35, 382-388 (2010).

30. Rinehart, M. T., Park, H. S. \& Wax, A. Influence of defocus on quantitative analysis of microscopic objects and individual cells with digital holography. Biomed. Opt. Express 6, 2067-2075 (2015).

31. Mohanty, J., Nagababu, E. \& Rifkind, J. M. Red blood cell oxidative stress impairs oxygen delivery and induces red blood cell aging. Front. Physiol. 5, 84 (2014).

32. Clark, M. R., Mohandas, N., Caggiano, V. \& Shohet, S. B. Effects of abnormal cation transport on deformability of desiccytes. J. Supramol. Struct. 8, 521-532 (1978).

33. Alshalani, A., Howell, A. \& Acker, J. P. Impact of blood manufacturing and donor characteristics on membrane water permeability and in vitro quality parameters during hypothermic storage of red blood cells. Cryobiology $\mathbf{8 0}$, 30-37 (2018).

34. Klein, H. G. \& Anstee, D. J. Mollison's Blood Transfusion in Clinical Medicine (John Wiley \& Sons, 2014)

35. Park, $H$. et al. Measuring cell surface area and deformability of individual human red blood cells over blood storage using quantitative phase imaging. Sci. Rep. 6, 34257 (2016).

36. Franco, R. S. The measurement and importance of red cell survival. Am. J. Hematol. 84, 109-114 (2009).

37. d'Onofrio, G. et al. Simultaneous measurement of reticulocyte and red blood cell indices in healthy subjects and patients with microcytic and macrocytic anemia. Blood 85, 818-823 (1995).

38. Franco, R. S. et al. Changes in the properties of normal human red blood cells during in vivo aging. Am. J. Hematol. 88, 44-51 (2013).

39. Gifford, S. C., Derganc, J., Shevkoplyas, S. S., Yoshida, T. \& Bitensky, M. W. A detailed study of time-dependent changes in human red blood cells: from reticulocyte maturation to erythrocyte senescence. Br. J. Haematol. 135, 395-404 (2006).

40. Gambhire, P. et al. High aspect ratio sub-micrometer channels using wet etching: application to the dynamics of red blood cell transiting through biomimetic splenic slits. Small 13, 1700967 (2017). 